\title{
OTIOTOMICS
}

Revista de economía, empresa y sociedad

Dossier «Prevención de riesgos laborales: tendencias en tiempo de crisis»

UNA NUEVA REALIDAD

\section{La difícil conciliación de la obligación empresarial de evaluar los riesgos con el teletrabajo}

\section{Mar Sabadell i Bosch}

Profesora de la Universitat Oberta de Catalunya (UOC). Estudios de Economía y Empresa

\section{Guillermo García González-Gastro}

Profesor de la Universidad Internacional de La Rioja (UNIR). Facultad de Ciencias Jurídicas, Sociales y Humanidades

RESUMEN El paradigma de salud laboral implementado con la Ley de Prevención de Riesgos Laborales en 1995 está impulsando nuevos modelos de gestión preventiva, aún incipientes, que apuestan decididamente por las denominadas organizaciones saludables. Fruto del cambio operado, se ha dejado atrás la tradicional acepción de seguridad e higiene, vinculada esencialmente con los accidentes del trabajo y las enfermedades profesionales, para acoger un término de mucho mayor alcance, seguridad y salud, que comprende la salud del trabajador en toda su extensión, física, psíquica y social.

Tras veinte años de vigencia de la norma, muchos son los logros y avances conseguidos en materia preventiva, pero también son múltiples los retos que se presentan en los próximos años. Entre otros, los relacionados con la ruptura de las barreras espaciales y temporales de la actividad laboral, como consecuencia de la incorporación de las tecnologías de la información y la comunicación. En este sentido, el artículo aborda la problemática que se origina en la aplicación de la normativa de seguridad y salud laboral al teletrabajo.

El análisis se centra en presentar el problema desde la misma base de aplicación práctica de la obligación de seguridad y salud laboral al teletrabajo, la evaluación de riesgos laborales, que constituye el fundamento sobre el que se construye y enlaza todo nuestro andamiaje normativo en materia de prevención de riesgos laborales.

PALABRAS CLAVE teletrabajo; trabajo a distancia; evaluación de riesgos; seguridad y salud laboral; tecnologías de la información y la comunicación (TIC) 


\title{
The difficult reconciliation of the corporate responsibility to assess the risks with telework
}

\begin{abstract}
The occupational health paradigm implemented with the Law on Prevention of Occupational Risks in 1995 is promoting new models of preventive management, still incipient, in favor of the so-called healthy organizations. As a result of this change, there has been a move from the traditional concept of safety and health, essentially linked with occupational accidents and diseases, towards a more far-reaching concept, safety and health, including workers' health in its whole extent, whether physical, psychological and social.
\end{abstract}

After twenty years of the enforcement of the rule, there are many achievements and progress has been made in prevention, but many challenges will arise in the coming years. Among others, those related to the breakdown of space and time barriers in the labor activity as a result of the incorporation of ICT. In this regard, the article deals with the problem that arises in the enforcement of occupational health and safety in telework.

The present analysis focuses on the problem from the very basis of the practical application of the requirement of occupational safety and health in telework, the occupational risk assessment, which is the foundation on which all our regulatory structure on prevention of occupational hazards is built and connected.

KEYWORDS telework; home-base telework; telecommuting; remote working; risk prevention; health and safety; ICT

\section{Introducción}

En la actualidad, la existencia de estructuras productivas flexibles y de un alto grado de digitalización de la información, así como de los procesos para su gestión, permiten que el trabajo se pueda llevar a cabo de manera diferente, y que el trabajador se pueda conectar a la empresa mediante redes telemáticas externas desde cualquier ubicación y en cualquier momento. Si hace veinte años las posibilidades del teletrabajo eran todavía limitadas, hoy, la mejora de la conectividad, la generalización de los dispositivos móviles y el desarrollo de tecnologías colaborativas están facilitando la extensión de esta nueva forma de trabajar.

El teletrabajo se configura como una realidad muy diversa y compleja que se caracteriza por acercar el trabajo al trabajador, evitando que este deba desplazarse a la sede física del empleador. La diversidad con la que se presenta esta realidad en la práctica hace que resulte muy difícil, cuando no imposible, elaborar una definición unívoca que abarque las diversas manifestaciones con las que el teletrabajo se presenta en la sociedad actual. En todo caso, lo que sí cabe afirmar es que todas estas complejas realidades, que se engloban bajo el término teletrabajo, generan disfunciones en los clásicos conceptos sobre los que se ha venido cimentando tradicionalmente el derecho del trabajo, y evidencian la necesidad de realizar un proceso de adaptación o reinterpretación de las clásicas instituciones laborales, con el fin de cohonestarlas con las nuevas fórmulas de organización productiva basadas en el uso intensivo y extensivo de las tecnologías de la información y la comunicación.

A continuación se analiza someramente cuándo y cómo aplicar la Ley de Prevención de Riesgos Laborales (LPRL), en caso de teletrabajo, sin entrar en un análisis de los riesgos laborales, genéricos o particulares, vinculados a este tipo de prestación. La LPRL parte de la laboralidad de la prestación de servicios para proteger la seguridad y salud, y el teletrabajo entra dentro de su campo de protección cuando se realice bajo el paraguas de una relación laboral incluida en el ámbito de aplicación del Estatuto de los Trabajadores. Sin embargo, a 
pesar de la inclusión formal del teletrabajo por cuenta ajena en el ámbito de aplicación de la LPRL, el ordenamiento jurídico español deviene un marco difícilmente aplicable en la práctica del teletrabajo. Y ello, porque nuestro sistema preventivo se fundamenta en la evaluación de riesgos laborales como elemento nuclear de toda la obligación de seguridad, y esta evaluación encuentra en el teletrabajo múltiples obstáculos para ser desarrollada, lo que frustra, en último término, la eficacia del deber de seguridad empresarial y debilita el derecho a la seguridad y salud laboral del teletrabajador.

\section{1995-2015: veinte años de la Ley de Prevención de Riesgos Laborales. Logros y retos de futuro}

Resulta indudable que el mayor éxito de la LPRL ha sido constituirse como motor de cambio de nuestra concepción de la seguridad y salud laboral, que, abandonando su dimensión reparadora y protectora, se ha consolidado como esencialmente preventiva. La LPRL, que transpone la directiva marco europea ${ }^{1}$, introduce en nuestro ordenamiento jurídico un nuevo modelo de gestión de la salud laboral, más acorde con las nuevas concepciones del derecho del trabajo y con nuestro sistema constitucional de derechos y libertades. Las empresas empiezan a valorar la necesidad de implicarse en el desarrollo de esta nueva dimensión de la salud, que va más allá de las lesiones fácilmente observables, y a efectuar una relectura de los riesgos a los que se encuentra sometido el trabajador. Frente a la lesión que se produce de forma visible en el lugar de trabajo, asociada inmediatamente al riesgo industrial, hoy adquiere más relevancia, e implica una mayor protección, la exposición a ciertos riesgos, vinculados a la propia evolución del lugar y organización del trabajo, capaces de lesionar la salud de una manera más paulatina, en procesos de prolongada latencia, que conducen al progresivo deterioro de la salud².

Sin embargo, la aplicación del nuevo modelo preventivo ha generado disfunciones en sus veinte años de vigencia; por ejemplo, la indeterminación de algunas obligaciones preventivas, como la formación o la vigilancia de la salud, las dificultades de integración efectiva de la prevención en las organizaciones productivas y el escaso papel que ha desempeñado la negociación colectiva en una materia tan esencial.

Entre todas ellas, merece especial mención la delimitación del ámbito de aplicación subjetivo de la normativa preventiva. La vocación de universalidad de la LPRL, aplicándose a la totalidad de los trabajadores por cuenta ajena y empleados públicos, tiene importantes excepciones en la práctica. En este sentido, se excluye explícitamente a algún colectivo de trabajadores por cuenta ajena, como los empleados del hogar, sin corregir dicha exclusión en las distintas normas posteriores que se han dictado³. Exclusión que también alcanza a los trabajadores autónomos, que solo son considerados por la LPRL en relación con la obligación de coordinación de actividades empresariales. Además, la LPRL no tiene en consideración determinadas prestaciones de servicios que, ajenas al ámbito jurídico-laboral, deben gozar de igual protección en materia de seguridad y salud ex. arts. 14, 15 y 40 de nuestra Constitución.

Junto a las exclusiones explícitas, la LPRL se muestra inoperante para poder dar respuesta a las nuevas formas de trabajo. Los años de vigencia de la LPRL han estado marcados por la emergencia y consolidación de las tecnologías de la información y la comunicación en los modelos organizativos y en las estructuras laborales. Asimismo, han coincidido con la paulatina digitalización y extensión de las tecnologías a los distintos ámbitos de la sociedad. Fruto de estos cambios, el teletrabajo va penetrando como una alternativa al trabajo presencial y constituye una modalidad de prestación cada vez más practicada.

La LPRL incluye dentro de su ámbito de aplicación a los teletrabajadores en los mismos términos que los trabajadores, y por tanto les resultan aplicables los derechos y las obligaciones preventivas contenidas en la ley y en

1. Directiva Marco 89/391/CEE.

2. Igartua (2008).

3. Vid. Real Decreto 1620/2011. 
las normas de desarrollo. Sin embargo, la aplicación de la legislación preventiva al ámbito del teletrabajo encuentra serias barreras culturales y jurídicas que hacen que en la práctica carezca de virtualidad. Y ello, porque el teletrabajo supone la irrupción de una nueva manera de trabajar en la que ciertos elementos clave del contrato de trabajo no se presentan en la forma tradicional.

\section{Teletrabajo y trabajo a distancia}

La primera cuestión que plantea la aplicación del derecho a la seguridad y salud laboral al teletrabajador está relacionada con la propia definición del teletrabajo. No existe una definición única de lo que es el teletrabajo, y establecer una delimitación clara y consistente de una realidad tan diversa no es fácil. Encontramos una variedad de formas de teletrabajo al igual que una pluralidad de términos, según el contexto donde se aplica. Teletrabajo como instrumento de conciliación, teletrabajo en un contexto de flexibilidad organizativa, teletrabajo como forma de descentralización productiva, teletrabajo como estrategia medioambiental o como fórmula de trabajo frente a situaciones excepcionales o desastres naturales, entre otros. Se van consolidando en la empresa fórmulas de trabajo flexible, e-trabajo, teletrabajo o trabajo remoto, sin olvidar vocablos más internacionales, como e-work, virtual work, homework, telework o telecommuting.

La reforma laboral de 2012 ha buscado promover nuevas formas de desarrollar la actividad laboral y «dar cabida al teletrabajo» en nuestro ordenamiento jurídico ${ }^{4}$. Sin embargo, el término teletrabajo solo se recoge en la Exposición de motivos de la reforma y, en su articulado, el legislador ha optado por incorporar de manera genérica el trabajo a distancia, sin referencia alguna al uso intensivo de las nuevas tecnologías, y eliminar el antiguo contrato de trabajo a domicilio ${ }^{5}$. La tímida reforma operada en esta cuestión (vid. artículo 13 del Estatuto de los trabajadores, introducido por la Ley 3/2012) no acomete con decisión una regulación del teletrabajo ni desde una perspectiva laboral ni desde la dimensión de la seguridad y salud laboral stricto sensu, y parece encuadrar el teletrabajo en un nuevo concepto jurídico: el trabajo a distancia.

La locución nominal trabajo a distancia constituye un calco semántico de la palabra teleworking, término anglosajón que designa al teletrabajo, pero esta identidad en la expresión utilizada no se traslada al plano conceptual. El trabajo a distancia se define legalmente como aquel que se realiza de manera preponderante en el domicilio del trabajador, o en el lugar libremente elegido por este, de modo alternativo a su desarrollo presencial en el centro de trabajo de la empresa ${ }^{6}$. Es precisamente la dimensión locativa la que caracteriza el trabajo a distancia, y no se hace depender su reconocimiento de la utilización de tecnologías digitales. En contraste, las taxonomías de teletrabajo que recoge la literatura internacional coinciden en caracterizar al trabajo a distancia a partir de dos dimensiones, la tecnológica y la locativa ${ }^{7}$. Y es que, de manera general, se admite que existe teletrabajo cuando las tecnologías de la información y la comunicación permiten al trabajador realizar la actividad lejos del lugar habitual de trabajo.

Contrariamente a lo imaginable, la reforma no condiciona el trabajo a distancia al uso de las TIC, y ello comporta que el encaje del teletrabajo en esta figura presente algunas dificultades. Ni todo trabajo a distancia puede considerarse teletrabajo, ni todo teletrabajo tiene la consideración de trabajo a distancia. Por un lado, encontramos que el trabajo que se realiza a distancia, sin que las tecnologías de la información y la comunicación lo posibiliten, no es teletrabajo. Por otro, el teletrabajo que se realiza de forma esporádica o puntual y limitado o residual en relación con la jornada de trabajo no constituye trabajo a distancia. Tampoco se reconoce como trabajo a distancia el teletrabajo que realiza el trabajador para complementar el trabajo presencial el fin de semana, por las noches o mientras se encuentra de viaje, más allá del horario y lugar de trabajo presencial.

4. Aprobada por el Gobierno mediante el Real Decreto-Ley 3/2012, de 10 de febrero, y refrendada por el Parlamento mediante la Ley 3/2013, de 6 de julio (en adelante Ley 3/2012).

5. Exposición de motivos y art. 6 de la Ley 3/2012.

6. Artículo 13 del Estatuto de los Trabajadores introducido por la Ley 3/2012.

7. Garret y Danziger (2007). 
El teletrabajo se considera trabajo a distancia cuando se lleva a cabo de manera preponderante en el domicilio del trabajador y de modo alternativo a la prestación presencial. También se considerará a distancia cuando el teletrabajo se lleva a cabo en otro lugar que haya elegido el trabajador. La conformación legal lleva a colegir que cuando la actividad contratada sea consustancialmente no presencial, el teletrabajo no puede considerarse a distancia al no constituirse en una alternativa real al trabajo presencial en el centro de trabajo. En síntesis, el concepto legal de trabajo a distancia no depende del uso de las TIC en el trabajo sino del tipo de actividad laboral realizada ${ }^{8}$.

Legalmente, cuando las condiciones en las que se lleva a cabo el teletrabajo permiten calificarlo de trabajo a distancia, el teletrabajador tiene derecho a una adecuada protección en materia de seguridad y salud laboral y se le aplican los mismos derechos que a los trabajadores presenciales, salvo aquellos que sean inherentes a la realización de la prestación laboral en el centro de trabajo de manera presencial. Quedaría fuera de esta garantía legal la obligación de seguridad en el teletrabajo cuando no pueda calificarse como trabajo a distancia. Además, la reforma reconoce explícitamente la equiparación retributiva de los trabajadores a distancia respecto a los presenciales, el ejercicio de los derechos de representación colectiva en los términos previstos en el Estatuto de los Trabajadores y los derechos a la formación y a la promoción del trabajador a distancia.

La aparente extensión de la aplicación de la normativa de seguridad y salud en el trabajo a los trabajadores a distancia es básicamente garantista, dado que, con anterioridad a la reforma, los teletrabajadores ya quedaban incluidos bajo la tutela de la LPRL y su normativa de desarrollo, por el principio de generalidad y no exclusión. Tras la reforma laboral, las dificultades de aplicación al teletrabajo de la normativa de protección en materia de seguridad y salud laboral siguen sin quedar resueltas y se mantiene la inseguridad jurídica en una cuestión demasiado trascendente. El legislador no dota de la esperable seguridad jurídica al teletrabajo para fomentar que las empresas apuesten sin reservas por flexibilizar la prestación de trabajo mediante este modelo organizativo.

\section{Teletrabajo y realidad empresarial}

El teletrabajo constituye una realidad dinámica cuya evolución va ligada al constante desarrollo tecnológico y que, de facto, va penetrando en las empresas y en los puestos de trabajo, aunque de manera desigual. La ausencia de una única definición de teletrabajo comporta que la cuantificación del fenómeno sea difícilmente comparable e incluso que existan cifras dispares. En España, la Encuesta sobre el uso de las tecnologías de la información y de las comunicaciones en las empresas del INE ${ }^{9}$ permite obtener dos indicadores relacionados con el teletrabajo. El primero se refiere a las empresas que disponen de empleados que trabajan fuera de los locales de la empresa de forma regular (datos desde 2005); y el segundo, a las empresas que permiten a sus trabajadores el acceso remoto a la empresa (datos desde 2009).

En los últimos años se observa, en primer lugar, un aumento progresivo del número de empresas que disponen de empleados que trabajan fuera de los locales de la empresa de manera regular, al menos media jornada semanal, y se conectan a los sistemas TIC de la organización mediante redes telemáticas externas.

8. Parece desprenderse que, cuando el trabajador por su actividad no desarrolle la prestación laboral de manera presencial en el centro de trabajo, como por ejemplo un transportista o un comercial, no podrá considerarse que realiza trabajo a distancia porque por la propia naturaleza de la actividad la prestación laboral no se puede prestar en el centro de trabajo.

9. Disponible en: http://www.ine.es/jaxi/menu.do?type=pcaxis\&path=/t09/e02\&file=inebase. 
Gráfico 1. Trabajo remoto

Porcentaje de empresas que disponen de teletrabajadores.

Evolución interanual (encuestas 2005-06 a 2012-13)

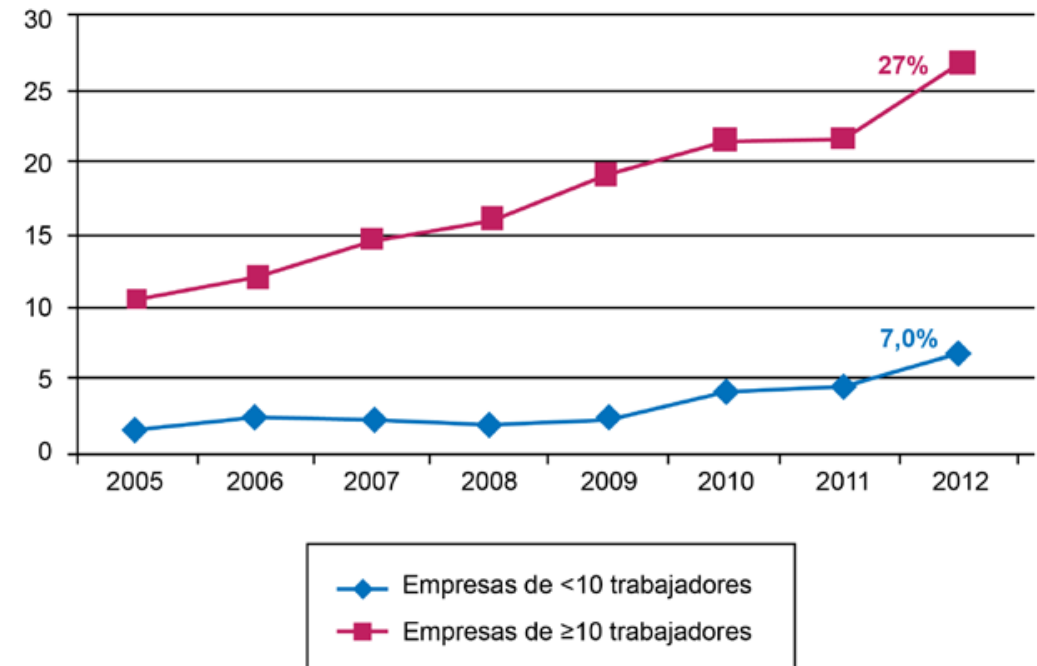

Fuente: Elaboración propia, a partir de los datos del INE.

La consolidación de un porcentaje significativo de empresas que reconoce que en sus plantillas tienen trabajadores que teletrabajan un mínimo de media jornada semanal es general, pero a medida que aumenta el tamaño de la empresa este porcentaje es superior. De promedio, en el año 2012, una de cada cuatro empresas aproximadamente reconocen tener teletrabajadores, trabajadores remotos, si bien la cifra varía en función del número de empleados de la organización.

\section{Gráfico 2. Trabajo remoto}

Distribución del teletrabajo, en función del tamaño de las empresas (según número de trabajadores)

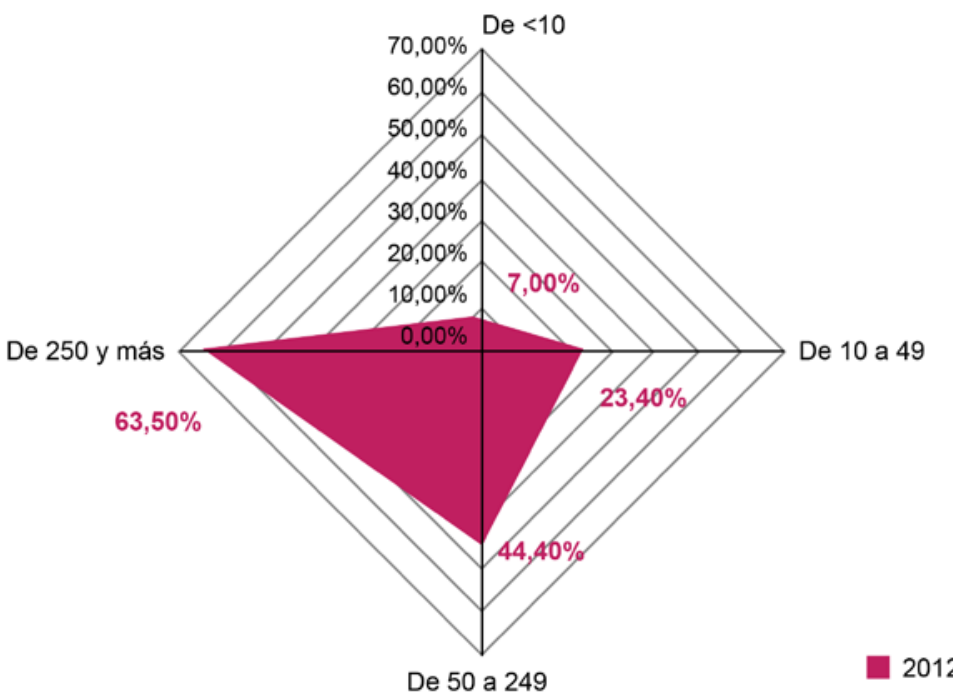

Fuente: Elaboración propia a partir de los datos del INE. 
A partir de 2013, la Encuesta de usos TIC no incluye el indicador de trabajo remoto, a pesar de la relevancia del dato. El cambio que recoge el cuestionario está relacionado, posiblemente, con el concepto de trabajo a distancia que ha establecido la reforma laboral de 2012. La dimensión tecnológica ha desaparecido del concepto legal de trabajo a distancia, mientras que el indicador de trabajo remoto incluye en su definición el uso de las TIC, en clara referencia al teletrabajo.

En relación con el segundo indicador, podemos señalar que el porcentaje de empresas que reconoce proporcionar a sus empleados acceso remoto al correo electrónico, a documentos de trabajo y a las aplicaciones de la empresa, mediante conexión a internet fija, inalámbrica o móvil es claramente mayoritario. Este porcentaje también está relacionado directamente con el tamaño de la empresa, de manera que el acceso remoto es más limitado en el caso de las microempresas. En 2012, frente al 27\% de empresas de más de diez trabajadores que reconocen disponer de teletrabajadores, el 65,5\% reconocen permitir que el trabajador pueda en algún momento teletrabajar, aunque ello no significa que lo haga ni de manera regular ni de manera formal. El hecho de proporcionar a los trabajadores el acceso remoto permite que estos se puedan conectar a la empresa mediante el uso de las TIC mientras se encuentran de viaje de trabajo, los fines de semana o fuera de los horarios laborales, para mejorar la conciliación vida-trabajo o en caso de baja laboral, entre otros.

\section{Gráfico 3. Acceso remoto}

Porcentaje de empresas, por tamaño, que facilitan a sus trabajadores acceso remoto a la empresa. Evolución interanual (encuestas 2010-11 a 2014-15)

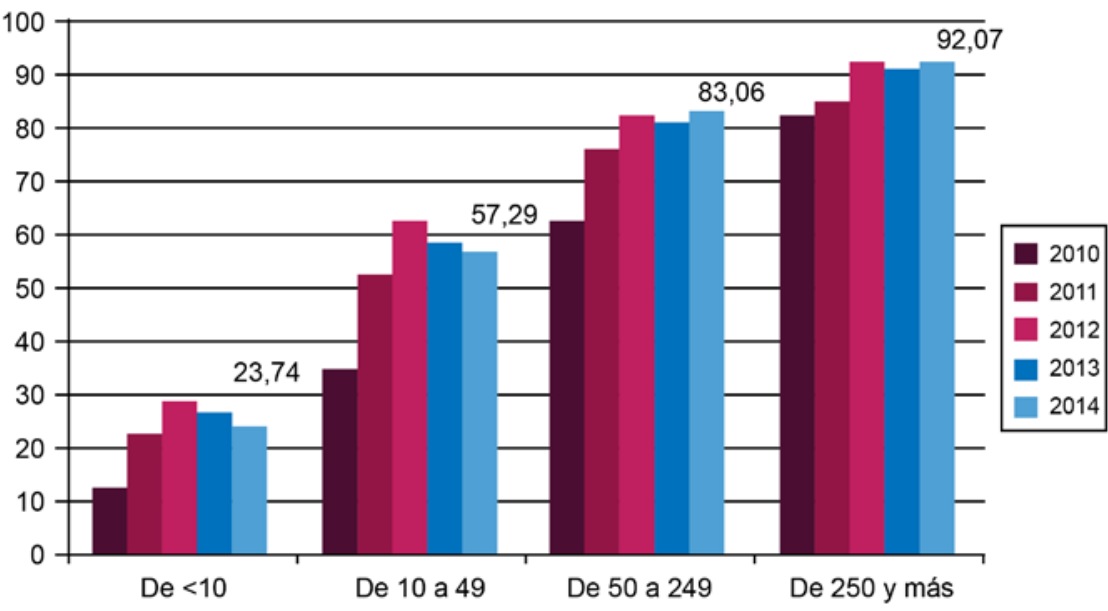

Fuente: Elaboración propia a partir de los datos del INE.

Sin embargo, en los últimos dos años se ha producido un estancamiento del indicador de acceso remoto, e incluso en las empresas de menos de cincuenta trabajadores la tendencia alcista se ha invertido. Curiosamente este cambio se registra coincidiendo con la primera encuesta elaborada con posterioridad a la reforma laboral de 2012. Es probable que este retroceso en el volumen de empresas que permiten a los trabajadores acceder de forma remota al correo, documentos y aplicaciones mediante redes externas, obedezca a las dificultades para dar cumplimiento efectivo al deber de seguridad y salud reconocido explícitamente para el trabajo a distancia con la reforma laboral. En principio, el derecho de seguridad y salud en el trabajo no puede venir limitado por las concretas circunstancias de organización y lugar en que se desarrolla la prestación de teletrabajo. El trabajador a distancia se encuentra dentro del ámbito de organización y dirección de la empresa y el empresario no pierde legalmente su poder de vigilancia y control sobre la prestación. No obstante, es evidente que encuentra indudables dificultades para responder de las condiciones en las que se ejecuta la prestación de teletrabajo. 


\title{
4. Teletrabajo y prevención. La evaluación de riesgos
}

La normativa de seguridad y salud en el trabajo parte del principio, al menos presuntivo, de que el empresario controla o puede controlar el espacio, medio y lugar donde se ejecuta la prestación ${ }^{10}$. Por ello se establece el deber del empresario de proteger a los trabajadores frente a los riesgos que se deriven de la prestación laboral y se reconoce el derecho de los trabajadores a una protección eficaz en materia de seguridad y salud en el trabajo. Se trata de un deber intransferible y el empresario no puede traspasar su obligación a otros sujetos implicados en la protección ${ }^{11}$, ni trasladar al trabajador el coste de las medidas que deban adoptarse ${ }^{12}$.

El empresario es el responsable del cumplimiento real y efectivo del deber de seguridad y salud y tiene que observar un comportamiento diligente para asegurar el efecto preventivo. Para ello, viene obligado a prever los riesgos, a adoptar cuantas medidas sean necesarias para una protección adecuada de los trabajadores y a adaptar progresivamente las medidas preventivas conforme a las nuevas circunstancias laborales y los nuevos mecanismos de producción ${ }^{13}$. Ello exige desarrollar una acción permanente de seguimiento de la actividad preventiva con el fin de perfeccionar de manera continua el nivel de protección existente.

Como parte del deber general de prevención, el empresario tiene una primera obligación que se concreta en calibrar la magnitud de los riesgos de su empresa. La evaluación de riesgos debe permitir al empresario obtener información necesaria para valorar la posibilidad de que el trabajador sufra un daño teniendo en cuenta las condiciones de trabajo. Para cada lugar de trabajo, el empresario debe realizar una evaluación inicial de los riesgos para la salud y la seguridad de los trabajadores, teniendo en cuenta sus características, y actualizarla cuando cambien las condiciones de trabajo, se introduzcan nuevas tecnologías o se modifique el acondicionamiento del lugar de trabajo ${ }^{14}$.

Por evaluación de riesgos hay que entender «el estudio de los potenciales riesgos para los trabajadores que el proceso productivo de una empresa puede comportar» ${ }^{15}$. La evaluación se referirá tanto a los riesgos generales que afecten a todos los trabajadores, por razón del tipo de actividad, como a los riesgos específicos a los que estén expuestos determinados trabajadores por las características de sus puestos de trabajo o por las características o estado de salud de la persona que lo ocupa. Se trata por tanto de una evaluación personalizada que debe analizar las condiciones de trabajo, tanto desde el punto de vista objetivo, del entorno, como subjetivo, de la persona.

El teletrabajo, tenga o no consideración legal de trabajo a distancia, supone una modificación individual de las condiciones de trabajo y la aparición de potenciales nuevos riesgos. Así, cuando la empresa contrata a un trabajador al que autoriza a teletrabajar, deberá evaluar los riesgos que se derivan de estas condiciones de trabajo. Es evidente que las circunstancias que envuelven algún tipo de teletrabajo, especialmente el teletrabajo móvil o el teletrabajo a domicilio, presentan claras dificultades para llevar a cabo la evaluación previa del lugar de trabajo y las revisiones periódicas de las instalaciones y los equipos, así como de dar cumplimiento al deber del empleador de ordenar y controlar las medidas de prevención. Sin embargo, la Ley 31/95 no contiene en su articulado previsión alguna sobre posibles limitaciones con las que el empresario se pueda ver afectado en el cumplimiento de su deber de protección, y se mantiene la obligación de seguridad empresarial en toda su extensión como si el trabajador estuviera en los locales de la empresa.

Al respecto, el Acuerdo marco europeo sobre teletrabajo firmado el 16 de julio de 2002 por los agentes sociales $^{16}$ indica que el empresario es responsable de la protección de la salud y de la seguridad profesional del teletrabajador conforme a la Directiva 89/391, así como a las directivas particulares, legislaciones nacionales y convenios colectivos pertinentes. El acuerdo establece la obligación del empresario de informar al teletrabajador

\author{
10. Thibault (2001). \\ 11. Artículo 5.2 y 3 de la Directiva Marco 89/391. \\ 12. Artículo 14.5 LPRL. \\ 13. Pérez de los Cobos (1991). \\ 14. Artículo 16 LPRL y artículo 3 del Real Decreto 39/1997 por el que se aprueba el Reglamento de los Servicios de Prevención (en \\ adelante, RSP) \\ 15. Sala (2011). \\ 16. CES, UNICE, UEAPME y CEEP.
}


de la política de la empresa en materia de salud y seguridad en el trabajo, en especial sobre las exigencias relativas a las pantallas de datos, y el deber del teletrabajador de aplicar correctamente estas políticas de seguridad. Asimismo, señala que la verificación de la correcta aplicación de las normas de seguridad y salud corresponde al empresario, a los representantes de los trabajadores y/o a las autoridades competentes, pero no indica cómo se llevará a cabo. En este sentido, se reconoce el derecho a acceder al lugar del teletrabajo, si bien, cuando este sea el domicilio del teletrabajador, el acceso estará sometido a notificación y consentimiento previo del trabajador.

La problemática que origina la necesidad del consentimiento del teletrabajador para evaluar su puesto de trabajo cuando este se encuentra en su propio domicilio ha llevado a algunas organizaciones productivas, aunque constituyen una excepción, a condicionar la autorización para teletrabajar a que el trabajador permita el acceso de los técnicos de prevención a su domicilio, lugar de trabajo, para llevar a cabo la evaluación inicial de riesgos. Sin embargo, el consentimiento inicial del trabajador como requisito necesario para teletrabajar en el propio domicilio no constituye una respuesta preventiva eficiente más allá de sus efectos inmediatos. Y ello porque, por un lado, va a ser necesaria una nueva autorización para cualquier verificación o reevaluación posterior y, por otro, porque el consentimiento inicial debe entenderse como esencialmente revocable al incidir sobre múltiples derechos fundamentales (singularmente, derecho a la intimidad e inviolabilidad del domicilio).

Otras organizaciones que tienen implantado el teletrabajo en su estructura productiva, con el fin de salvar las dificultades del acceso del personal técnico al puesto de trabajo del teletrabajador, han ido implantando un sistema de evaluación de riesgos laborales basado en la propia identificación y valoración de riesgos por el propio teletrabajador. Esta ha sido también la modalidad adoptada por la Administración pública para la implantación de programas de teletrabajo ${ }^{17}$. La autoevaluación de riesgos comporta que el teletrabajador autocomprueba su puesto de trabajo con pantallas de visualización de datos y adopta las medidas preventivas adecuadas. Para evaluar el puesto de trabajo, la empresa facilita una lista de autocomprobación e informa al teletrabajador sobre los riesgos relacionados con el medio ambiente de trabajo, los equipos de trabajo y los métodos de trabajo. Generalmente, el proceso se acompaña de formación e incluso puede capacitarlos para que adopten las medidas preventivas necesarias con el fin de eliminar o reducir los riesgos detectados.

Desde una perspectiva técnico-preventiva, la Nota Técnica de Prevención (NTP) número 412, «Teletrabajo: criterios para su implantación", publicada por el Instituto Nacional de Seguridad e Higiene en el Trabajo, aborda la cuestión del teletrabajo, ofreciendo un marco conceptual de este y proporcionando recomendaciones sobre las fases que deberían seguirse para su implantación. La NTP se centra, de entre las distintas posibilidades de localización, en el teletrabajo realizado en el domicilio del trabajador y recomienda seguir seis fases para una eficaz implantación. La segunda fase de desarrollo, relativa a criterios de selección de participantes y definición de procedimientos, hace referencia a la necesidad de identificar las necesidades de formación para su implantación, de definir los sistemas de apoyo para el teletrabajador, de establecer las necesidades de equipamiento, costes, servicios de mantenimiento, pólizas de seguro y sistemas de control y de prever los aspectos legales relacionados con cuestiones de seguridad e higiene, entre los que encontramos la evaluación de riesgos.

\section{Principales desajustes de la evaluación de riesgos en el teletrabajo}

Como se ha señalado, la obligación de seguridad empresarial se basa en nuestro ordenamiento jurídico en la evaluación de riesgos laborales, en cuanto a instrumento de gestión empresarial clave para el desarrollo y ejecución de las obligaciones preventivas posteriores (formación, información, vigilancia de la salud, entre otras). Pese a que formalmente la LPRL obliga al empresario a evaluar los riesgos laborales de sus trabajadores, con independencia del lugar en el que se presenten los servicios, lo cierto es que esta evaluación presenta, como ya se ha apuntado, numerosas dificultades para ser llevada a cabo de modo eficaz y completo en el caso de los teletrabajadores. Estos desajustes entre la obligación formal de seguridad y salud laboral (encarnada en la

17. Vid. Manual de Teletrabajo en www.seap.minhap.gob.es 
evaluación de los riesgos) y su cumplimiento material en el caso del teletrabajo se pueden sintetizar en cinco aspectos fundamentales:

- Derecho a la inviolabilidad del domicilio (art. 18.2 Constitución española). Los problemas que plantea la seguridad y salud en el teletrabajo son de máxima relevancia, especialmente cuando el teletrabajo se desarrolla en el propio domicilio del trabajador. La salvaguarda de los derechos a la inviolabilidad del domicilio y de respeto a la intimidad personal o familiar del trabajador impiden que el poder directivo y de organización que tiene el empresario en relación con el trabajo incluya una autorización a priori para acceder en cualquier momento al domicilio del teletrabajador, lugar de trabajo, para evaluar las condiciones en las que se desarrolla el teletrabajo y comprobar la efectividad y el cumplimiento de las medidas de protección. Además, aun en el caso de que el teletrabajador autorice al empresario a acceder a su domicilio para evaluar los riesgos asociados al teletrabajo, esta autorización no puede entenderse como general, de manera que no permite la acción preventiva continua que corresponde al empresario.

- Validez y eficacia de la autoevaluación. La validez y eficacia del sistema evaluativo basado en la autoevaluación es cuestionable teniendo en cuenta que el Reglamento de los Servicios de Prevención determina las capacidades y aptitudes necesarias para la evaluación de los riesgos ${ }^{18}$. En principio no parece que pueda responsabilizarse al trabajador de la evaluación de riesgos vía autoevaluación, dado que esta actuación tiene la consideración de función preventiva y el teletrabajador, en términos generales, carece de la necesaria formación para llevarla a cabo ${ }^{19}$.

Podría argüirse que con la formación de nivel básico del anexo IV del Real Decreto 39/1997, el teletrabajador podría estar en disposición de evaluar su propio puesto de trabajo. Sin embargo, no parece que pueda asociarse tal evaluación al nivel básico de funciones preventivas. Aunque prima facie pueda aparentar ser sencillo evaluar un puesto de teletrabajo, no cabe considerarla una evaluación elemental, si tenemos en cuenta, por poner un ejemplo, que las condiciones ambientales y lumínicas del teletrabajo deberían ser objeto de evaluación. Cuando la evaluación de riesgos exige el establecimiento de una estrategia de medición o una interpretación o aplicación no mecánica de los criterios de evaluación, constituye una actividad preventiva calificada de nivel superior ${ }^{20}$. Por ello, nos inclinamos a pensar que la evaluación corresponde a una función de nivel superior o, al menos, debe desarrollarse con el apoyo externo, real y efectivo, de un técnico capacitado para el desempeño de estas funciones.

Ello no es incompatible con fomentar sistemas de autoevaluación previos y complementarios que permitan al teletrabajador tomar conciencia de los riesgos asociados al teletrabajo y refuercen el seguimiento de las medidas preventivas para evitarlos o minimizarlos.

- Concepto de puesto de trabajo ad-hoc. Con el teletrabajo se está individualizando el lugar de realización de la prestación, y al mismo tiempo se va difuminando y reconfigurando de forma continua alrededor de criterios de movilidad. Dado que el teletrabajo móvil permite al teletrabajador trabajar en cualquier lugar y en todo momento, siempre y cuando se disponga de una tecnología digital y de la posibilidad de conectarse en red, está surgiendo un nuevo concepto de puesto de trabajo que carece de los elementos que han caracterizado el concepto industrial clásico. Además, el teletrabajo está muy vinculado con actividades intensivas en conocimiento y estas van adoptando un papel creciente en la economía. En este caso, el sistema de vigilancia de la salud, revisado e integrado, puede constituir una herramienta eficaz para prevenir unos riesgos no solo difícilmente evaluables sino incluso, en muchos casos, desconocidos.

18. Vid. artículos 35 c), 36 b) y 37 b) del RSP.

19. La formación mínima exigida para poder realizar una evaluación elemental debe tener una duración mínima de treinta horas y ajustar su contenido al anexo IV del RSP.

20. La capacitación para una evaluación superior requiere disponer de una titulación universitaria oficial y poseer una formación mínima acreditada, cuya duración no sea inferior a las seiscientas horas, con el contenido especificado en el anexo VI del RSP. 
- Delimitación de los riesgos que evaluar. Los mecanismos de autoevaluación o listas de chequeo se han centrado tradicionalmente en los riesgos asociados a los usuarios de pantallas visualización de datos. A través de los sistemas de autoevaluación implementados por teletrabajadores no debidamente cualificados, se corre el riesgo de adoptar una evaluación puramente formal, para dar cumplimiento a la normativa, sin realizar una valoración integral de riesgos. Se desconocen todavía los riesgos asociados al teletrabajo, por lo que resulta difícil anticiparse a estos, como demanda cualquier política preventiva, pero parece claro que los riegos psicosociales tienen un rol relevante. La evaluación de riesgos en el teletrabajo no debe limitarse a los aspectos ergonómicos ni al cumplimiento de las medidas preventivas derivadas del trabajo con pantallas de visualización de datos. Precisamente el teletrabajo comporta una nueva forma de organizar el trabajo al que se le pueden asociar riegos psicosociales relacionados con el uso de las tecnologías (como es el caso del tecnoestrés, la fatiga informativa y la tecnoadicción), pero también otros más específicos relacionados con la organización del tiempo, la confusión de rol, la disponibilidad permanente, la desvinculación de la organización, el aislamiento del trabajador y la pérdida de relación interpersonal. Las condiciones de trabajo en el teletrabajo demandan una profunda reflexión sobre los efectos de los condicionantes individuales y sus posibles repercusiones a medio y largo plazo sobre la salud.

- Determinación de la naturaleza común o profesional de la contingencia. En el caso del teletrabajador, resulta más difícil determinar la naturaleza común o profesional de las posibles lesiones o daños para la salud que pueda padecer. El hecho de utilizar una misma tecnología para relacionarnos en nuestra vida cotidiana y para trabajar está difuminando la frontera entre tiempo de trabajo y tiempo de no trabajo, lo que añade un grado de complejidad a la hora de determinar el origen, común o profesional, del daño y la consecuente responsabilidad para hacerle frente. Además, los riesgos asociados al uso de las tecnologías de la información y la comunicación (trastornos músculo-esqueléticos, trastornos visuales y oculares, seguridad estructural y tecnológica, adicción a las TIC, entre otros) no aparecen vinculados exclusivamente a las horas de trabajo, sino a la forma de vida de la sociedad actual, lo que incrementa su grado de incidentabilidad. Ello afecta directamente a los contornos y dintornos de la obligación de seguridad empresarial, que se presentan en el caso del teletrabajo de manera borrosa e imprecisa, lo que dificulta el cumplimiento efectivo del deber de prevención. Esta confusión se presenta de forma más intensa en el caso del teletrabajo, y resulta muy complicado discernir el origen laboral o extralaboral de determinados daños para la salud generados en los teletrabajadores por el uso de las TIC, lo que suscita problemas de delimitación en cuanto a la imputación de las responsabilidades que se pudieran derivar.

\section{Conclusiones}

La facultad del empresario de gestionar con flexibilidad interna la organización del trabajo, que es una manifestación de la libertad de empresa y de la defensa de la productividad reconocida en el artículo 38 de la Constitución española, ha de encontrar un equilibrio adecuado con el derecho a la seguridad de los trabajadores reconocido también en el artículo 40.2 del propio texto constitucional.

Como ha quedado evidenciado, la aplicación formal de la LPRL al teletrabajo presenta en su aplicación práctica numerosas disfunciones que dificultan el cumplimiento del deber de seguridad empresarial. Partiendo de la evaluación de riesgos laborales como la obligación principal, fundamento y núcleo de la obligación de seguridad y salud, se observa como su efectiva implementación en el ámbito del teletrabajo se encuentra con numerosos obstáculos que la pueden convertir en impracticable. Entre otros, el derecho a la inviolabilidad del domicilio, la reconfiguración móvil del lugar de trabajo y la normativa técnico-preventiva sobre competencias necesarias para realizar la evaluación de riesgos se configuran como tres serios impedimentos para que el empresario, en la práctica, pueda dar cumplimiento eficaz a su deber de seguridad en el caso de los teletrabajadores. 
Lo anteriormente expuesto no es incompatible con que al teletrabajador se le asignen determinadas funciones u obligaciones preventivas, deberes que por otra parte cabrían deducirse del art. 29 LPRL y que en el caso del teletrabajo adquieren una especial relevancia. En este sentido, la revisión previa del teletrabajador, sobre el cumplimiento de las disposiciones específicas en materia de seguridad y salud del trabajo desarrollado a distancia en su domicilio, puede ser un instrumento eficaz para una primera identificación de determinados riesgos laborales. Esta autoevaluación debería complementarse con un programa de formación adecuado que potenciara realmente la implicación de los teletrabajadores en el desarrollo de los sistemas de prevención del teletrabajo; y debería fomentar el análisis de los problemas de salud y seguridad vinculados al teletrabajo desde una perspectiva de la actividad real, para proponer intervenciones adaptadas a las necesidades y circunstancias específicas de cada empresa y propias de cada trabajador (convivencia de menores/ mayores a cargo en el domicilio, disponibilidad de espacio especifico para teletrabajar y estructuración de los descansos, desplazamientos largos con cambios horarios y trabajo en hoteles y aeropuertos, entre otros).

Además, no cabe obviar que la implicación de los teletrabajadores en el desarrollo de los sistemas de prevención del teletrabajo resulta vital para lograr las mejores soluciones preventivas, ya que son ellos quienes mejor conocen las características del propio puesto de trabajo. El teletrabajador posee la información y la experiencia necesarias para analizar los problemas desde la perspectiva más realista y puede proponer intervenciones adaptadas a las necesidades y circunstancias concretas de cada momento. Que los propios afectados participen en la identificación de los problemas y sus soluciones mejora, además, el clima de trabajo, aumenta la comunicación entre empresa-teletrabajador y contribuye a disminuir la sensación de aislamiento. Y es que no podemos olvidar que, en último término, y más allá de la óptica estrictamente jurídica, en un contexto donde el capital humano es el factor clave para la productividad y la creatividad de las organizaciones, la regeneración de las prácticas preventivas y la corresponsabilización para alcanzar óptimas condiciones de seguridad y salud en el trabajo deben constituir, en todo caso, un compromiso social de las empresas.

En síntesis, la solución al problema que plantea el teletrabajo en relación con la prevención de riesgos laborales no resulta fácil desde una perspectiva jurídica stricto sensu. Trascendiendo las estructuras normativas, la resolución de los problemas planteados debe venir de la conformación de modelos más participativos de protección de la seguridad y salud individual, promoviendo la participación de los distintos actores para la solución de los problemas que afectan al teletrabajo. La negociación colectiva puede aliviar la rigidez de la normativa preventiva, al mismo tiempo que ser un cauce adecuado para recopilar información y analizar y comprender mejor las condiciones de trabajo origen de la exposición a los riesgos. En último término, los desajustes que el teletrabajo presenta en relación con la seguridad y salud laboral solo obtendrán una solución definitiva con el desarrollo de un modelo sostenible de seguridad y salud en el teletrabajo, que pasa necesariamente por formar a todos los ciudadanos convenientemente en materia de prevención de riesgos asociados a la omnipresencia de las tecnologías de la información y la comunicación en nuestras vidas, de manera integral y transversal.

\section{Bibliografía}

BELZUNEGUI ERASO, A. (2002). Teletrabajo: estrategias de flexibilidad. Madrid: Consejo Económico y Social. (Colección Estudios; 130).

ESCUDERO RODRÍGUEZ, R. (2000). «Teletrabajo». En: Descentralización productiva y nuevas formas de organizativas del trabajo. X Congreso Nacional de Derecho del Trabajo y de la Seguridad Social. Zaragoza, 28 y 29 de mayo de 1999. Madrid: MTAS.

FERNÁNDEZ DOMÍNGUEZ, J. J. (2011). «Teletrabajo y prevención de riesgos laborales». En: La prevención de riesgos laborales y las nuevas formas de organización empresarial y del trabajo. II Jornadas universitarias castellano-leonesas. 
GARRET, R. K.; DANZIGER, J. N. (2007). «Which Telework? Defining and Testing a Taxonomy of TechnologyMediated Work at a Distance». Social Science Computer Review. Vol. 25, núm. 1, pág. 27-47.

IGARTUA MIRÓ, M. T. (2008). Sistema de prevención de Riesgos Laborales. Madrid: Tecnos.

INSTITUTO NACIONAL DE SEGURIDAD E HIGIENE EN EL TRABAJO (1996). «Teletreball: criteris per a la seva implantació». Nota Tècnica de Prevenció (NTP), núm. 412.

Ley 31/1995, de 8 de noviembre, de Prevención de Riesgos Laborales. Boletín Oficial del Estado, núm. 269 (10 de noviembre de 1995), pág. 32590-32611.

MARTÍNEZ LÓPEZ, F. J; RUIZ FRUTOS, C.; GARCÍA ORDAZ, M. (2008). «Teletrabajo: seguridad y salud sin importar la distancia». Revista Riesgos Laborales: Integración y desarrollo de la gestión de la prevención. Núm. 45.

MINISTERIO DE ADMINISTRACIONES PÚBLICAS (2006). «Manual para la implantación de programas piloto de teletrabajo en la Administración General del Estado». En: http://www.seap.minhap.gob.es/dms/es/web/areas/ funcion_publica/iniciativas/concilia/medidas/teletrabajo/Manual-Teletrabajo [Fecha de consulta: 15 de octubre de 2015].

PÉREZ DE LOS COBOS ORIHUEL, F. (1991). «La directiva marco sobre medidas de seguridad y salud de los trabajadores en el trabajo y la adaptación al ordenamiento (I)». Relaciones laborales. Núm. 8.

Real Decreto-Ley 3/2012, de 10 de febrero, de medidas urgentes para la reforma del mercado laboral. Boletín Oficial del Estado, núm. 36 (11 de febrero de 2012), pág. 12483-12546.

Real Decreto 39/1997, de 17 de enero, por el que se aprueba el Reglamento de los Servicios de Prevención. Boletín Oficial del Estado, núm. 27 (31 de enero de 1997), pág. 3031-3045.

SALA FRANCO, T. (2011). Derecho de la prevención de riesgos laborales. Valencia: Tirant lo Blanch.

THIBAULT ARANDA, J. (2001). El teletrabajo. Madrid: Consejo Económico y Social. (Colección Estudios; 88).

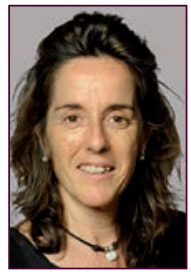

Mar Sabadell i Bosch

msabadell@uoc.edu

Profesora de la Universitat Oberta de Catalunya (UOC).

Estudios de Economía

y Empresa

Abogado, licenciada en Derecho por la Universidad de Barcelona y máster en Sociedad de la información y el conocimiento por la Universitat Oberta de Catalunya. Desde 1999 es profesora de los Estudios de Economía y Empresa de la UOC, donde imparte actualmente docencia, y durante los años 2006-2009 ha desarrollado el cargo de adjunta al vicerrector de Ordenación Académica y Profesorado.

Es miembro del grupo de investigación consolidado DigiBiz (Digital Business Research Group), adscrito al IN3. Sus intereses en investigación se centran actualmente en el estudio de la interrelación entre el uso de las TIC y el trabajo, y en el desarrollo de formas de trabajo flexible y trabajo a distancia, con una especial inquietud en materia de responsabilidad empresarial en la prevención de riesgos asociados al teletrabajo. 


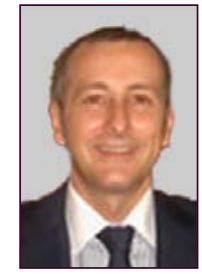

\section{Guillermo García González-Gastro} guillermo.garcia@unir.net Profesor de la Universidad Internacional de La Rioja (UNIR). Facultad de Ciencias Jurídicas, Sociales y Humanidades

Abogado y profesor agregado de Derecho del Trabajo en la Universidad Internacional de La Rioja. Doctor por la Universidad Autónoma de Barcelona y licenciado en Derecho, Ciencias Políticas y de la Administración y Ciencias del Trabajo. Ha impartido docencia en la Universitat Oberta de Catalunya, en la Universidad Autónoma de Barcelona y en la Universidad Miguel Hernández de Elche. Actualmente imparte docencia en exclusiva en la Universidad Internacional de La Rioja en las áreas de Derecho del Trabajo y Seguridad y Salud Laboral.

Es autor de múltiples manuales, obras colectivas y artículos especializados en materia de seguridad y salud laboral. Sus intereses de investigación se centran en la conformación jurídica de la seguridad y salud en el trabajo, el contenido de la obligación preventiva y las consecuencias derivadas de su incumplimiento.

Los textos publicados en esta revista están -si no se indica lo contrario- bajo una licencia Reconocimiento-Sin obras derivadas 3.0 España de Creative Commons. Puede copiarlos, distribuirlos y comunicarlos públicamente siempre que cite su autor y la revista y la institución que los publica (autoría, nombre de la revista, institución editora); no haga con ellos obras derivadas. La licencia completa se puede consultar en http://creativecommons.org/licenses/by-nd/3.0/es/deed.es.

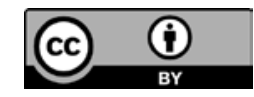

